Acta Crystallographica Section E

Structure Reports

Online

ISSN 1600-5368

\section{Sodium 3,5-dinitrobenzoate}

\section{Helen P. Jones, ${ }^{*}$ Amy L. Gillon‡ and Roger J. Davey}

Colloids, Crystals and Interfaces Group, School of Chemical Engineering and Analytical Sciences, The University of Manchester, PO Box 88, Manchester M60 1QD, England

\# Current address: Pharmaceutical R\&D, Pfizer Global R\&D (IPC 435), Ramsgate Road,

Sandwich, Kent CT13 9NJ, England

Correspondence e-mail:

h.jones-2@postgrad.manchester.ac.uk

\section{Key indicators}

Single-crystal X-ray study

$T=150 \mathrm{~K}$

Mean $\sigma(\mathrm{C}-\mathrm{C})=0.002 \AA$

$R$ factor $=0.025$

$w R$ factor $=0.066$

Data-to-parameter ratio $=7.3$

For details of how these key indicators were automatically derived from the article, see http://journals.iucr.org/e.
Sodium 3,5-dinitrobenzoate, $\mathrm{Na}^{+} \cdot \mathrm{C}_{7} \mathrm{H}_{3} \mathrm{~N}_{2} \mathrm{O}_{6}{ }^{-}$, was obtained by evaporation at room temperature of an aqueous solution of ethylenediammonium 3,5-dinitrobenzoate in sodium hydroxide. The structure is trigonal and the benzoate ion has twofold crystallographic symmetry.

\section{Comment}

During work on crystallization of the salt ethylenediammonium 3,5-dinitrobenzoate, an aqueous solution of the salt at $\mathrm{pH} 12$ was prepared and allowed to evaporate at room temperature, giving red prisms of sodium 3,5-dinitrobenzoate (NaDNB), (I). The crystal structure was not found in the Cambridge Structural Database (CSD, Version 5.25; Allen, 2002) and hence its structure was determined by single-crystal $\mathrm{X}$-ray diffraction at $150 \mathrm{~K}$.<smiles>[N][N+](=O)c1cc([N+](=O)[O-])cc([N+](=O)[O-])c1</smiles>

(I)

The benzoate ion is on a twofold axis of symmetry, passing through the carboxylate group (Fig. 1).

\section{Experimental}

3,5-Dinitrobenzoic acid (Aldrich, 99\%) was dissolved in sodium hydroxide solution and a solution of ethylenediamine (Aldrich, 99\%) was added. The solution was filtered and the $\mathrm{pH}$ recorded as 12.14. The solution $\mathrm{pH}$ was measured using an Accumet Basic AB15 pH

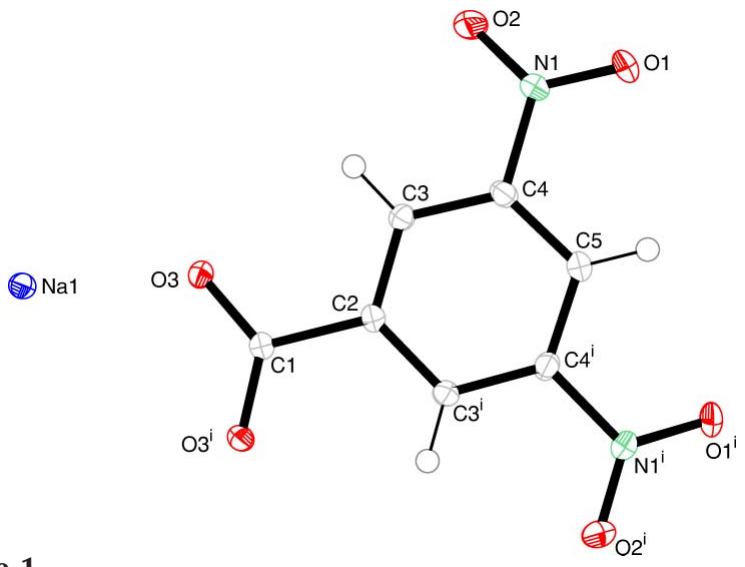

Figure 1

View of NaDNB, showing the whole benzoate anion. Displacement ellipsoids are drawn at the $50 \%$ probability level. [Symmetry code: (i) $x-y,-y,-z+\frac{2}{3}$.]
Received 5 April 2005 Accepted 10 May 2005 Online 14 May 2005
C 2005 International Union of Crystallography Printed in Great Britain - all rights reserved 


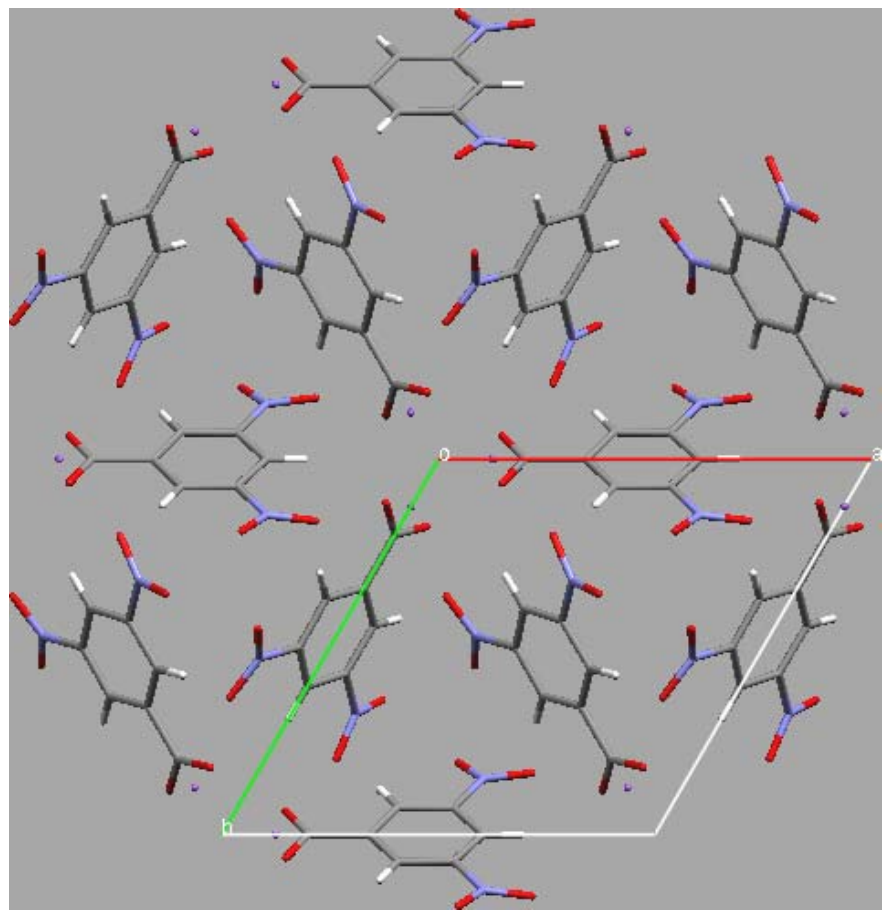

Figure 2

The packing of sodium 3,5-dinitrobenzoate, viewed along the $c$ axis, showing the threefold symmetry.

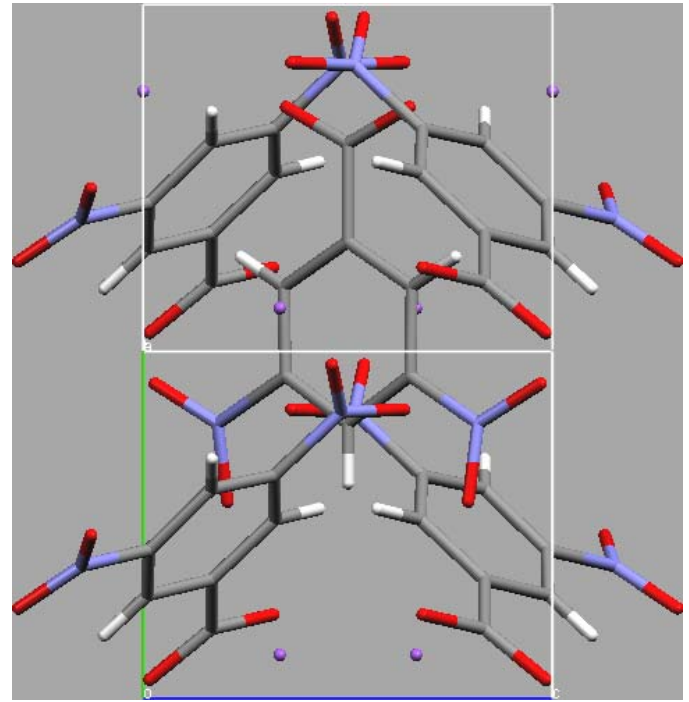

Figure 3

The twofold axis of symmetry perpendicular to the $c$ axis.

meter with an Accumet glass calomel pH electrode. The solution was allowed to evaporate to dryness in air at room temperature. Crystals of ethylenediammonium 3,5-dinitrobenzoate, sodium hydroxide and red prisms of sodium 3,5-dinitrobenzoate formed.

\section{Crystal data}

$\mathrm{Na}^{+} \cdot \mathrm{C}_{7} \mathrm{H}_{3} \mathrm{~N}_{2} \mathrm{O}_{6}{ }^{-}$

$M_{r}=234.1$

Trigonal, $P 3_{1} 21$

$a=10.7701(5) \AA$

$c=6.3526(2) \AA$

$V=638.15(5) \AA^{3}$

$Z=3$

$D_{x}=1.828 \mathrm{Mg} \mathrm{m}^{-3}$

Mo $K \alpha$ radiation

Cell parameters from 2522

reflections

$\theta=1.0-27.5^{\circ}$

$\mu=0.20 \mathrm{~mm}^{-1}$

$T=150 \mathrm{~K}$

Prism, red

$0.25 \times 0.25 \times 0.25 \mathrm{~mm}$

Data collection

Nonius KappaCCD diffractometer

Thick-slice $\varphi$ and $\omega$ scans

Absorption correction: multi-scan (Blessing, 1995)

$T_{\min }=0.796, T_{\max }=0.951$

3498 measured reflections

554 independent reflections

Refinement

Refinement on $F^{2}$

$R\left[F^{2}>2 \sigma\left(F^{2}\right)\right]=0.026$

$w R\left(F^{2}\right)=0.066$

$S=1.09$

554 reflections

76 parameters

$\mathrm{H}$-atom parameters constrained

$$
\begin{aligned}
& w=1 /\left[\sigma^{2}\left(F_{o}^{2}\right)+(0.0336 P)^{2}\right. \\
& \quad+0.1582 P] \\
& \quad \text { where } P=\left(F_{o}{ }^{2}+2 F_{c}^{2}\right) / 3 \\
& (\Delta / \sigma)_{\max }<0.001 \\
& \Delta \rho_{\max }=0.24 \mathrm{e}^{-3} \\
& \Delta \rho_{\min }=-0.18 \mathrm{e} \AA^{-3} \\
& \text { Extinction correction: SHELXL97 } \\
& \text { Extinction coefficient: } 0.14(2)
\end{aligned}
$$

In the absence of significant anomalous dispersion effects, Friedel pairs were merged. The choice of space group $P 3_{1} 21$ rather than $P 3_{2} 21$ is arbitrary. All $\mathrm{H}$ atoms were positioned geometrically and refined as riding, with $\mathrm{C}-\mathrm{H}=0.93-0.98 \AA$ and $U_{\text {iso }}(\mathrm{H})=1.2 U_{\text {eq }}(\mathrm{C})$.

Data collection: COLLECT (Nonius, 2000); cell refinement: SCALEPACK (Otwinowski \& Minor, 1997); data reduction: SORTAV (Blessing, 1987,1989, SCALEPACK and DENZO (Otwinowski \& Minor, 1997); program(s) used to solve structure: SHELXS97 (Sheldrick, 1997); program(s) used to refine structure: SHELXL97 (Sheldrick, 1997); molecular graphics: ORTEP-3 for Windows (Farrugia, 1997); software used to prepare material for publication: Win $G X$ (Farrugia, 1999).

The authors thank Sanofi-Aventis Ltd for funding.

\section{References}

Allen, F. H. (2002). Acta Cryst. B58, 380-388.

Blessing, R. H. (1987). Crystallogr. Rev. 1, 3-58.

Blessing, R. H. (1989). J. Appl. Cryst. 22, 396-397.

Blessing, R. H. (1995). Acta Cryst. A51, 33-38.

Farrugia, L. J. (1997). J. Appl. Cryst. 30, 565.

Farrugia, L. J. (1999). J. Appl. Cryst. 32, 837-838.

Nonius (2000). COLLECT. Nonius BV, Delft, The Netherlands.

Otwinowski, Z. \& Minor, W. (1997). Methods in Enzymology, Vol. 276, Macromolecular Crystallography, Part A, edited by C. W. Carter Jr \& R. M. Sweet, pp. 307-326. New York: Academic Press.

Sheldrick, G. M. (1997). SHELXS97 and SHELXL97. University of Göttingen, Germany. 


\section{supporting information}

Acta Cryst. (2005). E61, m1131-m1132［https://doi.org/10.1107/S1600536805014893]

Sodium 3,5-dinitrobenzoate

Helen P. Jones, Amy L. Gillon and Roger J. Davey

Sodium 3,5-dinitrobenzoate

Crystal data

$\mathrm{Na}^{+} \cdot \mathrm{C}_{7} \mathrm{H}_{3} \mathrm{~N}_{2} \mathrm{O}_{6}$

$M_{r}=234.1$

Trigonal, $P 3_{1} 21$

Hall symbol: P 31 2"

$a=10.7701(5) \AA$

$c=6.3526(2) \AA$

$V=638.15(5) \AA^{3}$

$Z=3$

$F(000)=354$

$D_{\mathrm{x}}=1.828 \mathrm{Mg} \mathrm{m}^{-3}$

Mo $K \alpha$ radiation, $\lambda=0.71073 \AA$

Cell parameters from 2522 reflections

$\theta=1.0-27.5^{\circ}$

$\mu=0.20 \mathrm{~mm}^{-1}$

$T=150 \mathrm{~K}$

Prism, red

$0.25 \times 0.25 \times 0.25 \mathrm{~mm}$

Data collection

Nonius KappaCCD diffractometer

Radiation source: Enraf Nonius FR590

Graphite monochromator

CCD rotation images, thick slices scans

Absorption correction: multi-scan

(Blessing, 1995)

$T_{\min }=0.796, T_{\max }=0.951$

3498 measured reflections

554 independent reflections

537 reflections with $I>2 \sigma(I)$

$R_{\text {int }}=0.027$

$\theta_{\max }=27.5^{\circ}, \theta_{\min }=3.8^{\circ}$

$h=-12 \rightarrow 13$

$k=-8 \rightarrow 13$

$l=-8 \rightarrow 8$

Refinement

Refinement on $F^{2}$

Least-squares matrix: full

$R\left[F^{2}>2 \sigma\left(F^{2}\right)\right]=0.026$

$w R\left(F^{2}\right)=0.066$

$S=1.09$

554 reflections

76 parameters

0 restraints

Primary atom site location: structure-invariant direct methods

Secondary atom site location: difference Fourier map

Hydrogen site location: inferred from neighbouring sites

$\mathrm{H}$-atom parameters constrained

$w=1 /\left[\sigma^{2}\left(F_{\mathrm{o}}^{2}\right)+(0.0336 P)^{2}+0.1582 P\right]$

where $P=\left(F_{\mathrm{o}}{ }^{2}+2 F_{\mathrm{c}}{ }^{2}\right) / 3$

$(\Delta / \sigma)_{\max }<0.001$

$\Delta \rho_{\max }=0.24 \mathrm{e} \AA^{-3}$

$\Delta \rho_{\min }=-0.18$ e $\AA^{-3}$

Extinction correction: SHELXL97, $\mathrm{Fc}^{*}=\mathrm{kFc}\left[1+0.001 \mathrm{xFc}^{2} \lambda^{3} / \sin (2 \theta)\right]^{-1 / 4}$

Extinction coefficient: 0.14 (2)

Special details

Geometry. All e.s.d.'s (except the e.s.d. in the dihedral angle between two 1.s. planes) are estimated using the full covariance matrix. The cell e.s.d.'s are taken into account individually in the estimation of e.s.d.'s in distances, angles and torsion angles; correlations between e.s.d.'s in cell parameters are only used when they are defined by crystal symmetry. An approximate (isotropic) treatment of cell e.s.d.'s is used for estimating e.s.d.'s involving 1.s. planes. 
Refinement. Refinement of $F^{2}$ against ALL reflections. The weighted $R$-factor $w R$ and goodness of fit $S$ are based on $F^{2}$, conventional $R$-factors $R$ are based on $F$, with $F$ set to zero for negative $F^{2}$. The threshold expression of $F^{2}>\sigma\left(F^{2}\right)$ is used only for calculating $R$-factors(gt) etc. and is not relevant to the choice of reflections for refinement. $R$-factors based on $F^{2}$ are statistically about twice as large as those based on $F$, and $R$ - factors based on ALL data will be even larger.

Fractional atomic coordinates and isotropic or equivalent isotropic displacement parameters $\left(\AA^{2}\right)$

\begin{tabular}{lllll}
\hline & $x$ & $y$ & $z$ & $U_{\text {iso }} * U_{\text {eq }}$ \\
\hline C1 & $0.2002(2)$ & 1 & -0.1667 & $0.0113(5)$ \\
C2 & $0.3418(2)$ & 1 & -0.1667 & $0.0122(5)$ \\
C3 & $0.3701(2)$ & $0.92732(19)$ & $-0.0104(2)$ & $0.0131(4)$ \\
H3 & 0.3033 & 0.8786 & 0.0952 & $0.016^{*}$ \\
O3 & $0.11648(14)$ & $0.93745(13)$ & $-0.01613(17)$ & $0.0138(3)$ \\
C4 & $0.4994(2)$ & $0.9287(2)$ & $-0.0143(2)$ & $0.0151(4)$ \\
C5 & $0.6034(2)$ & 1 & -0.1667 & $0.0155(5)$ \\
H5 & 0.6897 & 1 & -0.1667 & $0.019^{*}$ \\
N2 & $0.52972(17)$ & $0.85204(18)$ & $0.1513(2)$ & $0.0186(4)$ \\
Na1 & $0.87486(9)$ & $0.87486(9)$ & 0 & $0.0131(3)$ \\
O1 & $0.63430(15)$ & $0.83610(16)$ & $0.1274(2)$ & $0.0242(4)$ \\
O2 & $0.45083(19)$ & $0.8090(2)$ & $0.3043(2)$ & $0.0329(4)$ \\
\hline
\end{tabular}

Atomic displacement parameters $\left(\AA^{2}\right)$

\begin{tabular}{lllllll}
\hline & $U^{11}$ & $U^{22}$ & $U^{33}$ & $U^{12}$ & $U^{13}$ & $U^{23}$ \\
\hline $\mathrm{C} 1$ & $0.0111(8)$ & $0.0108(11)$ & $0.0118(10)$ & $0.0054(5)$ & $-0.0016(4)$ & $-0.0031(8)$ \\
$\mathrm{C} 2$ & $0.0115(9)$ & $0.0135(11)$ & $0.0122(10)$ & $0.0067(6)$ & $-0.0012(4)$ & $-0.0024(9)$ \\
$\mathrm{C} 3$ & $0.0136(9)$ & $0.0142(9)$ & $0.0121(8)$ & $0.0073(7)$ & $0.0010(6)$ & $0.0001(6)$ \\
$\mathrm{O} 3$ & $0.0118(6)$ & $0.0162(7)$ & $0.0133(6)$ & $0.0069(5)$ & $0.0013(4)$ & $0.0010(5)$ \\
$\mathrm{C} 4$ & $0.0170(8)$ & $0.0185(9)$ & $0.0132(8)$ & $0.0115(7)$ & $-0.0009(6)$ & $0.0006(7)$ \\
$\mathrm{C} 5$ & $0.0130(9)$ & $0.0179(13)$ & $0.0173(11)$ & $0.0089(6)$ & $0.0002(5)$ & $0.0005(9)$ \\
$\mathrm{N} 2$ & $0.0179(8)$ & $0.0226(9)$ & $0.0183(7)$ & $0.0125(7)$ & $0.0007(6)$ & $0.0056(6)$ \\
$\mathrm{Na} 1$ & $0.0131(4)$ & $0.0131(4)$ & $0.0128(4)$ & $0.0064(4)$ & $-0.00068(18)$ & $0.00068(18)$ \\
O1 & $0.0170(7)$ & $0.0323(9)$ & $0.0300(7)$ & $0.0174(7)$ & $0.0027(6)$ & $0.0104(6)$ \\
O2 & $0.0356(9)$ & $0.0548(11)$ & $0.0228(7)$ & $0.0335(9)$ & $0.0132(6)$ & $0.0209(7)$ \\
& & & & & &
\end{tabular}

Geometric parameters $\left(\AA,{ }^{o}\right)$

\begin{tabular}{|c|c|c|c|}
\hline $\mathrm{C} 1-\mathrm{O} 3$ & $1.2547(16)$ & $\mathrm{O} 3-\mathrm{Na} 1^{\mathrm{ii}}$ & $2.3416(14)$ \\
\hline $\mathrm{C} 1-\mathrm{C} 2$ & $1.525(3)$ & $\mathrm{C} 4-\mathrm{C} 5$ & $1.386(2)$ \\
\hline $\mathrm{C} 2-\mathrm{C} 3$ & $1.389(2)$ & $\mathrm{C} 4-\mathrm{N} 2$ & $1.471(2)$ \\
\hline $\mathrm{C} 3-\mathrm{C} 4$ & $1.386(2)$ & $\mathrm{C} 5-\mathrm{H} 5$ & 0.93 \\
\hline $\mathrm{C} 3-\mathrm{H} 3$ & 0.93 & $\mathrm{~N} 2-\mathrm{O} 2$ & $1.220(2)$ \\
\hline $\mathrm{O} 3-\mathrm{Na} 1^{\mathrm{i}}$ & $2.3083(11)$ & $\mathrm{N} 2-\mathrm{O} 1$ & $1.231(2)$ \\
\hline $\mathrm{O} 3^{\mathrm{iii}-\mathrm{C} 1-\mathrm{O} 3}$ & $126.5(2)$ & $\mathrm{O}^{\mathrm{iv}}-\mathrm{Na} 1-\mathrm{O}^{\mathrm{v}}$ & $167.89(8)$ \\
\hline $\mathrm{O} 3-\mathrm{C} 1-\mathrm{C} 2$ & $116.77(11)$ & $\mathrm{O} 3^{\mathrm{v}}-\mathrm{Na} 1-\mathrm{O}^{\mathrm{vi}}$ & $86.31(5)$ \\
\hline $\mathrm{C} 3^{\mathrm{iii}-\mathrm{C} 2-\mathrm{C} 3}$ & $119.9(2)$ & $\mathrm{O} 3^{\mathrm{v}}-\mathrm{Na} 1-\mathrm{O} 3^{\mathrm{vii}}$ & $102.24(5)$ \\
\hline $\mathrm{C} 3-\mathrm{C} 2-\mathrm{C} 1$ & $120.06(11)$ & 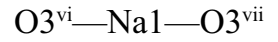 & $91.20(7)$ \\
\hline
\end{tabular}




$\begin{array}{llll}\mathrm{C} 4-\mathrm{C} 3-\mathrm{C} 2 & 118.90(16) & \mathrm{O}^{\mathrm{iv}}-\mathrm{Na} 1-\mathrm{O} 1 & 79.55(5) \\ \mathrm{C} 4-\mathrm{C} 3-\mathrm{H} 3 & 120.5 & \mathrm{O}^{\mathrm{v}}-\mathrm{Na} 1-\mathrm{O} 1 & 93.24(5) \\ \mathrm{C} 2-\mathrm{C} 3-\mathrm{H} 3 & 120.5 & \mathrm{O}^{\mathrm{vi}}-\mathrm{Na} 1-\mathrm{O} 1 & 82.64(5) \\ \mathrm{C} 1-\mathrm{O} 3-\mathrm{Na}^{\mathrm{i}} & 131.53(9) & \mathrm{O}^{\mathrm{vii}}-\mathrm{Na} 1-\mathrm{O} 1 & 162.96(5) \\ \mathrm{C} 1-\mathrm{O} 3-\mathrm{Na} 1^{\mathrm{ii}} & 125.81(12) & \mathrm{O}^{\mathrm{viii}}-\mathrm{Na} 1-\mathrm{O} 1 & 107.43(8) \\ \mathrm{Na} 1^{\mathrm{i}}-\mathrm{O} 3-\mathrm{Na} 1^{\mathrm{ii}} & 85.34(5) & \mathrm{O}^{\mathrm{i}}-\mathrm{Na} 1-\mathrm{Na} 1^{\mathrm{ix}} & 47.77(3) \\ \mathrm{C} 3-\mathrm{C} 4-\mathrm{C} 5 & 123.23(16) & \mathrm{O}^{\mathrm{v}}-\mathrm{Na} 1-\mathrm{Na} 1^{\mathrm{ix}} & 143.85(5) \\ \mathrm{C} 3-\mathrm{C} 4-\mathrm{N} 2 & 119.02(15) & \mathrm{O}^{\mathrm{vi}}-\mathrm{Na} 1-\mathrm{Na} 1^{\mathrm{ix}} & 77.64(3) \\ \mathrm{C} 5-\mathrm{C} 4-\mathrm{N} 2 & 117.74(16) & \mathrm{O}^{\mathrm{vii}}-\mathrm{Na} 1-\mathrm{Na} 1^{\mathrm{ix}} & 46.88(4) \\ \mathrm{C} 4^{\mathrm{iii}}-\mathrm{C} 5-\mathrm{C} 4 & 115.8(2) & \mathrm{O} 1^{\mathrm{viii}}-\mathrm{Na} 1-\mathrm{Na} 1^{\mathrm{ix}} & 108.62(3) \\ \mathrm{C} 4-\mathrm{C} 5-\mathrm{H} 5 & 122.1 & \mathrm{O} 1-\mathrm{Na} 1-\mathrm{Na} 1^{\mathrm{ix}} & 116.10(3) \\ \mathrm{O} 2-\mathrm{N} 2-\mathrm{O} 1 & 123.91(16) & \mathrm{Na} 1^{\mathrm{ix}}-\mathrm{Na} 1-\mathrm{Na} 1^{\mathrm{x}} & 100.20(3) \\ \mathrm{O} 2-\mathrm{N} 2-\mathrm{C} 4 & 118.34(14) & \mathrm{N} 2-\mathrm{O} 1-\mathrm{Na} 1 & 160.96(12) \\ \mathrm{O} 1-\mathrm{N} 2-\mathrm{C} 4 & 117.74(15) & & \end{array}$

Symmetry codes: (i) $-y+1, x-y+1, z+1 / 3$; (ii) $x-1, y, z$; (iii) $x-y+1,-y+2,-z-1 / 3$; (iv) $-x+1,-x+y,-z+1 / 3$; (v) $-x+y,-x+1, z-1 / 3$; (vi) $y, x+1,-z$; (vii) $x+1, y, z$; (viii) $y, x,-z$; (ix) $-y+2, x-y+1, z+1 / 3$; (x) $-x+y+1,-x+2, z-1 / 3$. 